\title{
A case of acute urinary retention after cesarean section caused by herpes zoster infection ${ }^{*}$
}

\author{
Atsushi Yoshida ${ }^{\#}$, Kenji Ishii, Keiko Saito, Isao Azuma \\ Department of Obstetrics and Gynecology, Nishisaitama-Chuo National Hospital, Tokorozawa, Japan \\ Email: " yoshida.atsushi@nifty.ne.jp
}

Received 12 May 2012; revised 15 June 2012; accepted 30 June 2012

\begin{abstract}
We experienced a case of acute urinary retention after an elective cesarean section. In this case, an epidural catheter was inserted for the postoperative pain relief, and we had to rule out anesthetic complications including epidural hematoma. After careful investigation, sacral herpes zoster was found to be responsible for urinary retention. Early administration of antiviral agent was started and the outcome was good. As various factors may lead to postpartum urinary disorders, we should be careful not to miss serious complications.
\end{abstract}

Keywords: Anesthesia; Cesarean Section; Herpes Zoster; Postpartum; Urinary Retention

\section{INTRODUCTION}

Postpartum urinary disturbance is a relatively common complication, which may be caused by a variety of factors. Urinary retention after cesarean section can be caused by surgical or anesthetic procedures and prompt response is necessary because delay in the diagnosis and therapy may result in severe aftereffects. Herpes zoster is one of the diseases which may cause acute urinary retention, and the clinical course is strongly affected by the timely initiation of the therapy. We here present a case of acute urinary retention as a chief complaint after cesarean section caused by herpes zoster infection.

\section{CASE REPORT}

The patient was a 40-year-old Japanese woman, para 1-0-2-2. Her height was $154 \mathrm{~cm}$ and pregestatinoal weight was $41 \mathrm{~kg}$ (body mass index was 17.3). Her past history was remarkable in that she had a cesarean section in her previous twin pregnancy. The patient also stated that she had had 2 spontaneous abortions in the first tri-

\footnotetext{
Disclosure: None of the authors have any conflicts of interest associated with this study.

${ }^{\#}$ Corresponding author.
}

mester.

Repeat cesarean section was scheduled at 38 weeks and 4 days. Preoperative laboratory data showed no particular problems (Table 1). Cesarean section was carried out as scheduled. Spinal anesthesia was administered successfully at the L 3 - 4 interspace using $0.5 \%$ hyperbaric bupivacaine $1.8 \mathrm{~mL}$ plus fentanyl $10 \mu \mathrm{g}$. Prior to the spinal anesthesia, an epidural catheter was inserted at the L 1 - 2 interspace for the postoperative pain relief. Total blood loss including amniotic fluid was $856 \mathrm{ml}$ and cesarean section was successfully performed uneventfully. A transverse skin incision was made on the old Pfannenstiel scar. A transverse incision was made into the anterior wall of the lower uterine segment. The patient was delivered of a 2886 gram boy infant in good condition. After the surgery, $0.2 \%$ ropivacaine hydrochloride was continuously given from the epidural catheter. On the second postoperative day, the epidural catheter was removed and no remarkable abnormal findings were noted by inspection or subjective symptoms. Heparin calcium of 5000 units was subcutaneously given on the evening of the surgery, on the morning of first postoperative day

Table 1. Preoperative laboratory data.

\begin{tabular}{ccccccccc}
\hline WBC & $\left(\times 10^{3}\right)$ & 6.9 & PT & $(\mathrm{sec})$ & 9.6 & TP & $(\mathrm{g} / \mathrm{dl})$ & 5.8 \\
$\mathrm{RBC}$ & $\left(\times 10^{4}\right)$ & 398 & APTT & $(\mathrm{sec})$ & 27.1 & $\mathrm{Alb}$ & $(\mathrm{g} / \mathrm{dl})$ & 3.5 \\
$\mathrm{Hb}$ & $(\mathrm{g} / \mathrm{dl})$ & 12.0 & & & & $\mathrm{GOT}$ & $(\mathrm{IU} / \mathrm{l})$ & 12 \\
$\mathrm{Ht}$ & $(\%)$ & 36.3 & & & & $\mathrm{GPT}$ & $(\mathrm{IU} / \mathrm{l})$ & 9 \\
$\mathrm{Plt}$ & $\left(\times 10^{4}\right)$ & 19.3 & & & $\mathrm{LDH}$ & $(\mathrm{IU} / \mathrm{l})$ & 155 \\
& & & & & $\mathrm{CPK}$ & $(\mathrm{IU} / \mathrm{l})$ & 33 \\
& & & & & $\mathrm{BUN}$ & $(\mathrm{mg} / \mathrm{dl})$ & 6.3 \\
& & & & & $\mathrm{Creat}$ & $(\mathrm{mg} / \mathrm{dl})$ & 0.5 \\
& & & & $\mathrm{Na}$ & $(\mathrm{mmol} / \mathrm{l})$ & 138 \\
& & & & & $\mathrm{~K}$ & $(\mathrm{mmol} / \mathrm{l})$ & 4.1 \\
& & & & & $\mathrm{Cl}$ & $(\mathrm{mmol} / \mathrm{l})$ & 104 \\
\hline
\end{tabular}


and the evening, and on morning of the second postoperative day.

Her postoperative course was uneventful until the 6th postoperative day. The patient complained of difficulty in urination and hypesthesia on her right lower abdomen and the medial surface of her right thigh. On the 7th postoperative day, the urethral catheterization was necessary because of urinary retention. The urethral catheterization was carried out 5 times on the 7th postoperative day and the total volume of catheterized urination was $2300 \mathrm{~mL}$. We suspected that the urinary retention might be caused by an epidural hematoma as a complication of the epidural catheterization. Magnetic resonance imaging of the spine revealed no abnormal mass which might be compressing the spinal cord (Figure 1). We consulted the anesthesiologists and they did not think the urinary retention as a complication of anesthetic procedures. On the 7th postoperative day we found hypochromic erythema on the skin of the patient's right sacral distribution (Figure 2). On the 8th postoperative day, the patient was examined by a dermatologist and she was diagnosed as having herpes zoster. Serum antibodies of varicella zoster virus was markedly elevated with $\mathrm{IgG}$ index at 128 (positive $\geq 2$ ) and IgM index 1.87 (positive $\geq 0.80$ ) and we considered that urinary retention had been caused by herpes zoster. The patient complained of slight pain but later of the sacral lesion accompanied by numerous vesicles, some of which broke down to form ulcerations. Gentamicin sulfate ointment was applied locally and aciclovir was given intravenously $(250 \mathrm{mg} \times$ 3 per day) for 7 days, followed by mecobalamin $500 \mu \mathrm{g}$ $\times 3$ per day orally. Because the urinary retention contin-

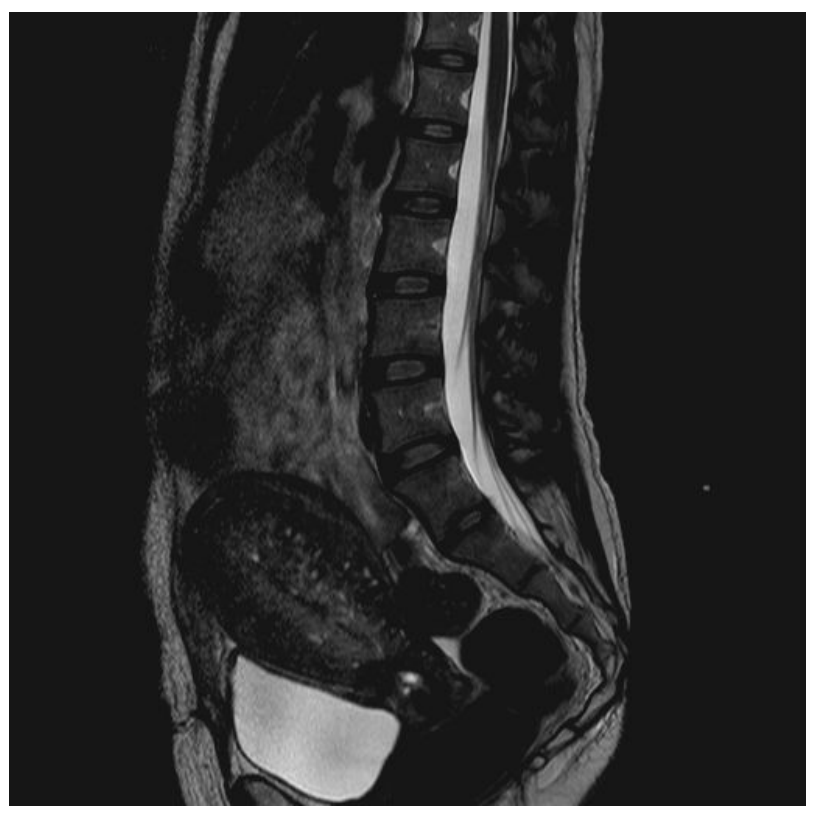

Figure 1. Spinal MRI of this case. No abnormal mass such as epidural hematoma was noted.

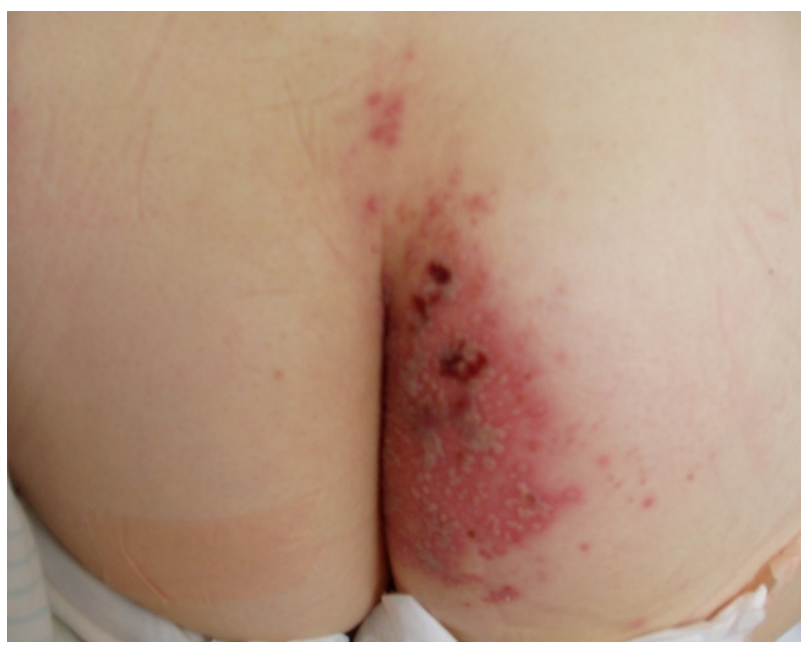

Figure 2. Hypochromic erythema on the skin of the patient's right sacral distribution.

ued, we consulted the urologists, who recommended inserting of an indwelling catheter. However, the patient chose intermittent self catheterization was shown how to do it.

On the 16th postoperative day, although the patient could not void, she chose to be discharged from the hospital and her self catheterization was continued. After the discharge, urinary retention improved gradually and on the 29th postoperative day the patient became able to urinate. The sacral lesion improved and mecobalamin oral administration was stopped. No recurrence of urinary retention was noted.

\section{DISCUSSION}

Postpartum urinary disturbance is a relatively common complication. After vaginal delivery, difficulty in urination or urinary retention often occurs as a result of carious factors such as fetal head compression on the peripheral nerve during labor, paralyzed urinary bladder, urethral edema or abdominal muscle relaxation. These urinary disturbances after vaginal delivery usually improve shortly. Postoperative urinary disturbance after cesarean section is also a relatively common occurrence, which may be affected by various factors such as postpartum bladder dysfunction and complications by surgical or anesthetic procedures [1-3]. It is reported that 1) multiple pregnancy, 2) lack of progress in labor, 3) low maternal body mass index and 4) use of postoperative analgesia are the risk factors for postpartum urinary retention after cesarean delivery $[2,4,5]$. Therefore, this case was thought to be high risk for postpartum urinary retention because it meets the factors (3) and (4) described above. The incidence of postpartum urinary retention associated with postoperative analgesia was reported to be affected by the analgesics and administration 
methods $[4,6]$. Epidural analgesia with morphine was thought to increase post-cesarean urinary retention [6].

Not only cesarean section, but also post surgical epidural analgesia is often complicated by urinary retention, which usually disappears shortly after the discontinuance of analgesics [7]. Therefore, it was thought to be unlikely that urinary retention in this case was directly caused by epidural analgesia. On the other hand, inserting or removing epidural catheter may cause an epidural hematoma, which can lead to urinary retention. Epidural hematoma caused by epidural catheter is usually associated with bleeding tendency in patients with complications such as coagulopathy, liver disease or anticoagulant medications. In our hospital, subcutaneous injection of heparin is used for preventing postoperative venous thrombosis in almost all cesarean cases but in the heparin administered cases, the epidural catheter is removed at least 4 hours after heparin injection. In this case of postoperative heparin injection the epidural catheter was removed after 5 hours of last heparin. Therefore, the possibility of an epidural hematoma caused by removal of the epidural catheter was not thought to be strong, but an epidural hematoma formation in a patient ordinary without bleeding tendency is reported [8]. Epidural hematoma is usually thought to be formed shortly after insertion or removal of epidural catheter, but the formation of epidural hematoma 9 days after removal of the epidural catheter is reported [9]. In our case, urinary retention occurring 6 days after the insertion and 4 days after the removal of the epidural catheter could not readily rule out the relationship between urinary retention and hematoma by the epidural catheter. Spinal MRI was obtained immediately, but no epidural hematoma or other mass which might be compressing the spinal nerves was noted.

Herpes zoster is an infection caused by varicella zoster virus. The primary infection with varicella zoster virus develops as varicella (chicken pox) and results in inflammatory lesion of the sensory root ganglions, meninges or spinal cord. After the primary infection, the virus enters the latent phase and remains dormant indefinitely. The reactivation and proliferation of the virus may recur as herpes zoster. It is reported that herpes zoster is reactivated by various factors such as advanced age, physical or psychological stress, general fatigue, trauma, common cold or impaired immunity in patients on steroids, malignant tumors or anticancer drugs [10]. In our current case, the patient was suspected to be at high risk for herpes zoster because she was in the postoperative period with fatigue and physical and psychological stresses. Usually herpes zoster is complicated by sever pain along the innervations, although in this case the patient complained little pain. Gluteal cutaneous lesion in this case was considered to be innervation area of the sacral nerve. Urinary retention is a common complica- tion of zoster [11]. Sacral herpes zoster is thought to induce detrusor areflexia by an inflammatory reaction, which begins in the dorsal root ganglia and spreads to the sacral segments of the spinal cord, and causes urinary retention as the result, but it is reported that lumber or thoracic zoster may cause urinary retention [12]. Sacral herpes may also cause rectal disorders, but in this case her rectal function was intact. In the therapy of zoster, prompt use of antiviral agents is very important. It is preferable to start antiviral agents within 72 hours of cutaneous-lesion outbreak. In this case, accurate time of onset of the erythema was unclear, but we could start aciclovir within two days of urinary retention, which was thought to be relatively early initiation of antiviral therapy. In the drug package insert (in this case we used VICCLOX I.V. Infusion $250 \mathrm{mg}$ by Kobayashi Kako Co. Ltd. Japan), concerning the use of aciclovir during the lactation period, it is written that "nursing mothers should discontinue breast feeding during treatment (transfer of this drug into breast milk has been reported)." However, Japan Drug Information Institute in Pregnancy (JDIP) in National Center for Child Health and Development announces that use of aciclovir is thought to be safe during nursing period. In our case, we explained to the patient the product document and the announcement by JDIP. After obtaining the informed consent, nursing was continued and no particular problems were noted.

\section{ACKNOWLEDGEMENTS}

I am deeply grateful to Dr. Mitsunao Kobayashi who provided helpful comments and suggestions.

\section{REFERENCES}

[1] Kermans, G., Wyndaele, J.J., Thiery, M. and DeSy, W. (1986) Puerperal urinary retention. Acta Urologica Belgica, 54, 376-385.

[2] Evron, S., Samueloff, A., Simon, A., Drenger, B. and Magora, F. (1985) Urinary function during epidural analgesia with methadone and morphine in post-cesarean section patients. Pain, 23, 135-144. doi:10.1016/0304-3959(85)90055-7

[3] Dray, A. and Metsch, R. (1984) Inhibition of urinary bladder contractions by a spinal action of morphine and other opioids. Journal of Pharmacology and Experimental Therapeutics, 231, 254-260.

[4] Chai, A.T., Wong, T., Mak, H.L.J., Cheon, C., Yip, S.K. and Wong, A.S.M. (2008) Prevalence and associated risk factors of retention of urine after cesarean section. International Urogynecology Journal, 19, 537-542. doi:10.1007/s00192-007-0470-1

[5] Liang, C.C., Tseng, L.H., Horng, S.G., Lin, I.W. and Chang, S.D. (2007) Correlation of pelvic organ prolapse quantification system scores with obstetric parameters and lower urinary tract symptoms in primiparae postpar- 
tum. International Urogynecology Journal, 18, 537-541. doi:10.1007/s00192-006-0195-6

[6] Liang, C.C., Chang, S.D., Wong, S.Y., Chang, Y.L. and Cheng, P.J. (2010) Effects of postoperative analgesia on postpartum urinary retention in women undergoing cesarean delivery. Journal of Obstetrics and Gynecology Research, 36, 991-995. doi:10.1111/j.1447-0756.2010.01252.x

[7] Shadle, B., Barbaro, C., Waxman, K., Connor, S. and Von Dollen, K. (2009) Predictors of postoperative urinary retention. American Surgeon, 75, 922-924.

[8] Chung, J.H., Hwang, J., Cha, S.C., Jung, T. and Woo, S.C. (2011) Epidural hematoma occurred by massive bleeding intraoperatively in cesarean section after combined spinal epidural anesthesia-A case report. Korean Journal of Anesthesiology, 61, 336-340. doi:10.4097/kjae.2011.61.4.336
[9] Guffey, P.J., Mckay, W.R. and Mckay, R.E. (2010) Epidural hematoma nine days after removal of a labor epidural catheter. Anesthesia \& Analgesia, 111, 992-995.

[10] Eshleman, E., Shahzad, A. and Cohrs, R.J. (2011) Varicella zoster virus latency. Future Virology, 6, 341-355. doi: $10.2217 /$ fvl.10.90

[11] Yamanishi, T., Yasuda, K., Sakakibara, R., Hattori, T., Uchiyama, T., Minamide, M. and Ito, H. (1998) Urinary retention due to herpes virus infections. Neurourology and Urodynamics, 17, 613-619. doi:10.1002/(SICI)1520-6777(1998)17:6<613::AID-NA $\mathrm{U} 5>3.0 . \mathrm{CO} ; 2-2$

[12] Julia, J.J. and Cholhan, H.J. (2007) Herpes zoster-associated acute urinary retention: A case report. International Urogynecology Journal, 18, 103-104. doi:10.1007/s00192-006-0066-1 